\title{
Paper-based piezoresistive MEMS sensors
}

\section{Citation}

Liu, Xinyu, Martin Mwangi, XiuJun Li, Michael O'Brien, and George M. Whitesides. 2011. “PaperBased Piezoresistive MEMS Sensors." Lab on a Chip 11, no. 13: 2189-2196.

\section{Published Version}

doi:10.1039/C1LC20161A

\section{Permanent link}

http://nrs.harvard.edu/urn-3:HUL.InstRepos:12967808

\section{Terms of Use}

This article was downloaded from Harvard University's DASH repository, and is made available under the terms and conditions applicable to Open Access Policy Articles, as set forth at http:// nrs.harvard.edu/urn-3:HUL.InstRepos:dash.current.terms-of-use\#OAP

\section{Share Your Story}

The Harvard community has made this article openly available.

Please share how this access benefits you. Submit a story.

\section{Accessibility}




\section{Paper-Based Piezoresistive MEMS Sensors}

Xinyu Liu ${ }^{1}$, Martin Mwangi ${ }^{1}$, XiuJun $\mathrm{Li}^{1}$, Michael O’Brien ${ }^{1}$, and George M. Whitesides ${ }^{1,2^{*}}$

${ }^{1}$ Department of Chemistry and Chemical Biology, ${ }^{2}$ Wyss Institute for Biologically Inspired Engineering, Harvard University, Cambridge, MA 02138, U.S.A.

*Corresponding author E-mail: gwhitesides@gmwgroup.harvard.edu 


\begin{abstract}
This paper describes the development of MEMS force sensors constructed using paper as the structural material. The working principle on which these paper-based sensors are based is the piezoresistive effect generated by conductive materials patterned on a paper substrate. The device is inexpensive ( $\$ 0.04 /$ device for materials $)$, simple to fabricate, light weight, and disposable. Paper can be readily folded into three-dimensional structures to increase the stiffness of the sensor while keeping it light in weight. The entire fabrication process can be completed within one hour without expensive cleanroom facilities using simple tools (e.g., a paper cutter and a painting knife). We demonstrated that the paper-based sensor can measure forces with moderate performance (i.e., resolution: $120 \mu \mathrm{N}$, measurement range: $\pm 16 \mathrm{mN}$, and sensitivity: $0.84 \mathrm{mV} / \mathrm{mN}$ ), and we applied this sensor to characterizing the mechanical properties of a soft material. Leveraging the same sensing concept, we also developed a paper-based balance with a measurement range of $15 \mathrm{~g}$, and a resolution of $390 \mathrm{mg}$.
\end{abstract}




\section{Introduction}

The past three decades have witnessed the extensive development of MEMS (Micro-ElectroMechanical Systems) devices and systems, which have found important applications in industry and medicine. ${ }^{1-3}$ Examples of commercially successful MEMS products include digital micromirror devices (DMD, Texas Instruments), accelerometers for triggering air bags in automobiles (Analog Devices and Motorola), and pressure/flow sensors for industrial uses (Honeywell).

Silicon-based materials (e.g., single crystal silicon, polycrystalline silicon, silicon dioxide, and silicon nitride) are the primary materials used for constructing MEMS devices, and manufacturing approaches are derived from microfabrication processes developed for integrated circuits (ICs). ${ }^{4}$ The microfabrication process as used for silicon-based MEMS (especially for prototyping) is time-consuming (days for a single batch) and requires access to cleanroom equipment. Both materials and use of cleanroom are expensive; and although the performance of silicon-based MEMS can be excellent, their relatively high cost has limited the applications they can address.

We are interested in the development of new MEMS technologies, where the emphasis is on minimizing cost, and the ratio of performance to cost is maximized by minimizing cost rather than maximizing performance. As the material to serve as the basis for this exploratory progress, we have chosen paper. A conceptually related effort to reduce the cost of diagnostic systems by developing paper-based diagnostic systems has developed into a new approach to diagnostic technologies. ${ }^{5-9}$ Paper is readily available, lightweight, and easy-to-manufacture; the paper substrate makes integration of electrical signal-processing circuits onto the paper-based MEMS devices straightforward. As our first investigation of paper for the construction of MEMS, we 
developed a paper-based pizoresistive force sensor and applied it to mechanical characterization of soft materials. We also demonstrated a paper-based weighing balance.

\section{Experimental design}

\section{Working principle of the paper-based force sensors}

The sensing principle of the paper-based force sensor is the piezoresistive effect of conductive materials patterned on a paper structure (a cantilever beam in this work). Many MEMS sensors (including commercial devices) also take advantage of the piezoresistive effect, but they are typically constructed from silicon-based semiconductor materials. Instead, we used paper as a structural material for construction of the devices; paper is much cheaper (e.g., $\$ 0.1 / \mathrm{m}^{2}$ for printing paper) than silicon, and provides other advantages (e.g., light weight, disposability, and ease of manufacturing) over silicon.

Fig. 1A shows a schematic diagram of a simple, paper-based force-sensing cantilever. In this device, a carbon resistor is located at the root of the cantilever. When a force is applied to the beam structure, the resistor experiences a mechanical strain/stress, which then induces a change in resistance of the resistor. Measuring the change in resistance allows quantification of the applied force.

\section{Device fabrication}

Fig. 1B summarizes the process used to fabricate the device. We fabricated paper cantilever beams by cutting $3 \mathrm{MM}$ chromatography paper (340 $\mu \mathrm{m}$ thick) using a laser cutter. We screenprinted carbon resistors using high-resistivity graphite ink, and contact pads using low-resistivity sliver ink. Fabricating an array of paper-based force sensors (Fig. 1C) typically takes less than one hour. 
Fig. 1 Paper-based piezoresistive force sensor. (A) Schematic view of a paper-based force sensor using a carbon resistor as the sensing component. (B) Fabrication process of the paper-based sensor involving laser cutting of paper and screen printing of carbon and silver inks. (C) A photograph of an array of four devices with labeled dimensions. 
Fig. 1

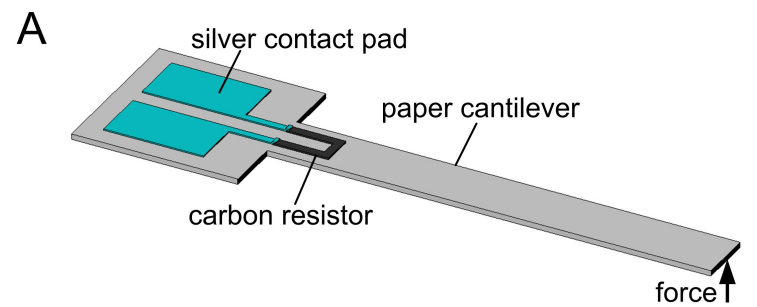

B (a) Laser pattern paper cantilever paper

(b) Screen print carbon resistor

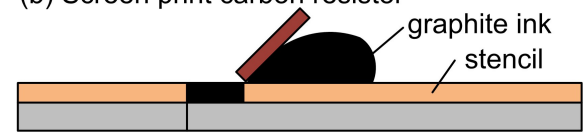

(c) Remove stencil and dry

(d) Screen print silver contact pad

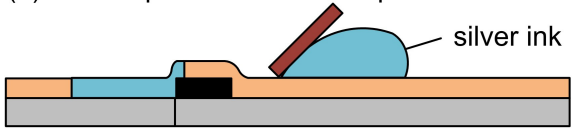

(e) Remove stencil and dry

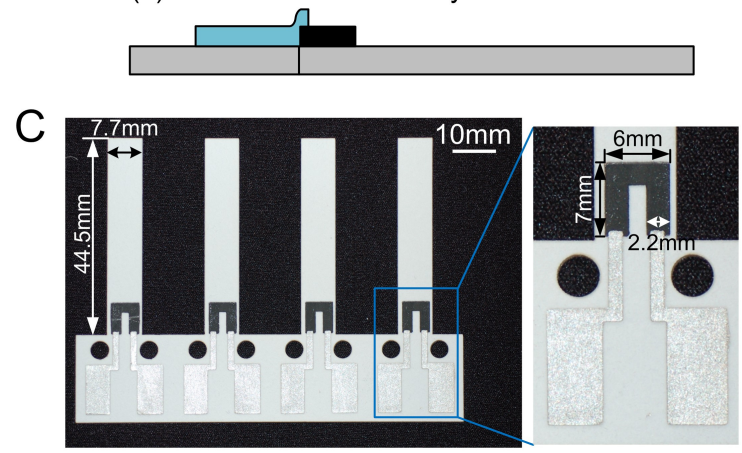


Paper is hydrophilic, and adsorption of water, with resultant changes in mechanical and electrical properties of the sensor, is a potential concern. To render the paper hydrophobic, we functionalized the surface hydroxyl groups of the paper with (tridecafluoro-1,1,2,2tetrahydrooctyl) trichlorosilane vapor to form surface silanol linkages, and thus generated a fluorinated, highly textured, hydrophobic surface (which is effectively superhydrophobic). This surface treatment minimizes the effect of environmental humidity on the mechanical and electrical properties of the sensor. The contact angle of water on the silanized paper substrate was $140^{\circ}$ (for comparison, contact angles of water on polyethylene and Teflon films are $100^{\circ}$ and $120^{\circ}$ respectively ${ }^{10,11}$ ).

\section{Results and discussion}

\section{Mechanical properties of paper cantilever beams}

We first characterized the stiffness of the paper cantilever beams using a precision balance (Fig. S1, force measurement resolution: $0.98 \mu \mathrm{N}$ ), by measuring forces applied to the free end of a paper cantilever as a function of the beam deflections. Fig. S2A shows force-deflection data based on measurements of seven devices. The stiffness of a paper cantilever $(44.5 \mathrm{~mm}$ long, 7.7 $\mathrm{mm}$ wide, and $0.34 \mathrm{~mm}$ thick) was determined to be $2.0 \pm 0.16 \mathrm{mN} / \mathrm{mm}$ (mean \pm one standard deviation).

We also tested the mechanical reliability of the paper device through reciprocating bending of a cantilever beam up to 1,000 times. After every 200 times of reciprocating bending, we tested the force-deformation curves of the paper cantilever seven times, and calculated the beam stiffness based on the data from the measurements. Fig. S2B shows data characterizing beam stiffness as a function of the number of bends. The change of the beam stiffness during the 1,000 cycles of bending is $<4 \%$. These data demonstrate stable mechanical properties (stiffness) of the 
paper cantilever beams, and suggest that (hydrophobized) paper is a suitable structural material for constructing MEMS devices.

To compare the stiffness of paper with that of other materials for constructing MEMS devices, we estimated the Young's modulus of the paper beam, based on the data of forcedeformation curves (Fig. S2A). We calculated the Young's modulus of the paper using a beam equation (Eq. 1), with the assumption that paper is a solid and homogeneous material:

$$
E=\frac{4 F L^{3}}{\delta W H^{3}}
$$

Here, $E$ is the Young's modulus (in $\mathrm{kPa}$ ) of the paper, $F$ is the force (in $\mathrm{mN}$ ) applied to the free end of paper beam, $\delta$ is the deflection (in mm) of paper beam, and $L, W$, and $H$ are length, width, and thickness (in mm) of the beam respectively. The Young's modulus of the paper was determined to be $2.0 \pm 0.17 \mathrm{GPa}(\mathrm{N}=7)$, which is $66 \sim 86$ times lower than that of silicon (130-170 GPa for single crystal silicon). ${ }^{4}$ We are aware that, due to the porous structure of the paper, the value of Young's modulus that we calculated is not the Young's modulus of cellulose itself (fibers of which form the paper), but an equivalent approximation to Young's modulus of the paper in the format of a porous sheet.

\section{Electrical properties of carbon resistors}

We measured the current-voltage (I-V) characteristic of the carbon resistor using a source meter (Keithley 2400). All the measured resistors $(\mathrm{N}=7)$ revealed a linear, ohmic I-V behavior (Fig. S3A), suggesting that the piezoresistive effect of the carbon resistor is mainly caused by the strain-induced shape deformations of the resistor. The resistance of the resistors we tested was $600 \pm 190 \Omega(\mathrm{N}=7)$.

We also investigated the temperature dependence of the carbon resistors by quantifying the temperature coefficient of resistance. This coefficient is defined as the ratio of relative change in 
resistance of a resistor $\left(\Delta R / R_{0}\right)$ to the change in temperature $(\Delta T)$. Fig. S3B shows the relative change in resistance of the carbon resistors as a function of the change in temperature. These data yield a temperature coefficient of resistance of $0.0012 \pm 0.0007 /{ }^{\circ} \mathrm{C}(\mathrm{N}=7)$.

\section{Sensor calibration}

We calibrated the paper-based force sensors using a precision balance (model EP64, Ohaus Explorer Pro) and a LCR (L: inductance, C: capacitance, and R: resistance) meter (model 885, BK Precision). Fig. 2A shows the experimental output of the sensor (i.e., the change in resistance) as a function of the input to the sensor (i.e., the force applied to the free end of a paper beam) when the carbon resistor was under compressive strain. The resistance changes linearly with the applied force. Based on the calibration curve (Fig. 2A), the range of force measurement was determined to be $\pm 16 \mathrm{mN}$, and the resolution of force measurement (detection limit) was 0.35 $\mathrm{mN}$ (corresponding to the detection limit of the LCR meter: $0.1 \Omega$ ). The sensitivity of the sensor (i.e., the slope of the curve of linear regression in Fig. 2A) was $0.29 \Omega / \mathrm{mN}$. With the current experimental system, the resolution of force measurement was primarily limited by the resolution of the resistance measurements $(0.1 \Omega)$ obtained using the LCR meter. Using a highresolution LCR meter, or integrating a signal-processing circuit to read the change in resistance more accurately, would increase the resolution of force measurement.

Interestingly, the force sensors showed a nonlinear response (Fig. S4) when the carbon resistor was stretched rather than compressed. Second-order polynomial equations fitted the experimental data well $\left(\mathrm{R}^{2}>0.99\right)$. The cause of the nonlinear behavior of the carbon resistor under tensile strain still remains unclear. Since a linear sensor response is desired in most force sensing applications, we opted only to test and use the paper-based sensor in the mode that places the carbon resistor under compressive strain (which we call 'compressive mode'). All the 
Fig. 2 Calibration of the paper-based force sensor when the carbon resistor is under compression strain. The Error bars in all figures represent one standard deviation. (A) Calibration plot of the output of the sensor (resistance change) as a function of the input to the sensor (applied force). The solid lines represent a linear fit to the experimental data with a regression equation: (A) $y=0.27 x\left(R^{2}=0.998, N=7\right)$. The slope of the solid line represents the sensitivity of the sensors. (B) Calibration plots of the relative change in resistance as a function of the applied strain. The solid lines represent a linear fit to the experimental data with a regression equation: $y=4.1 \times\left(R^{2}=0.998\right.$, $\mathrm{N}=7$ ). The slope of the solid line represents the gauge factor of the sensor, which is defined as the ratio of relative chance in resistance $\left(\Delta \mathrm{R} / \mathrm{R}_{0}\right)$ to the applied mechanical strain $(\varepsilon)$. 
Fig. 2
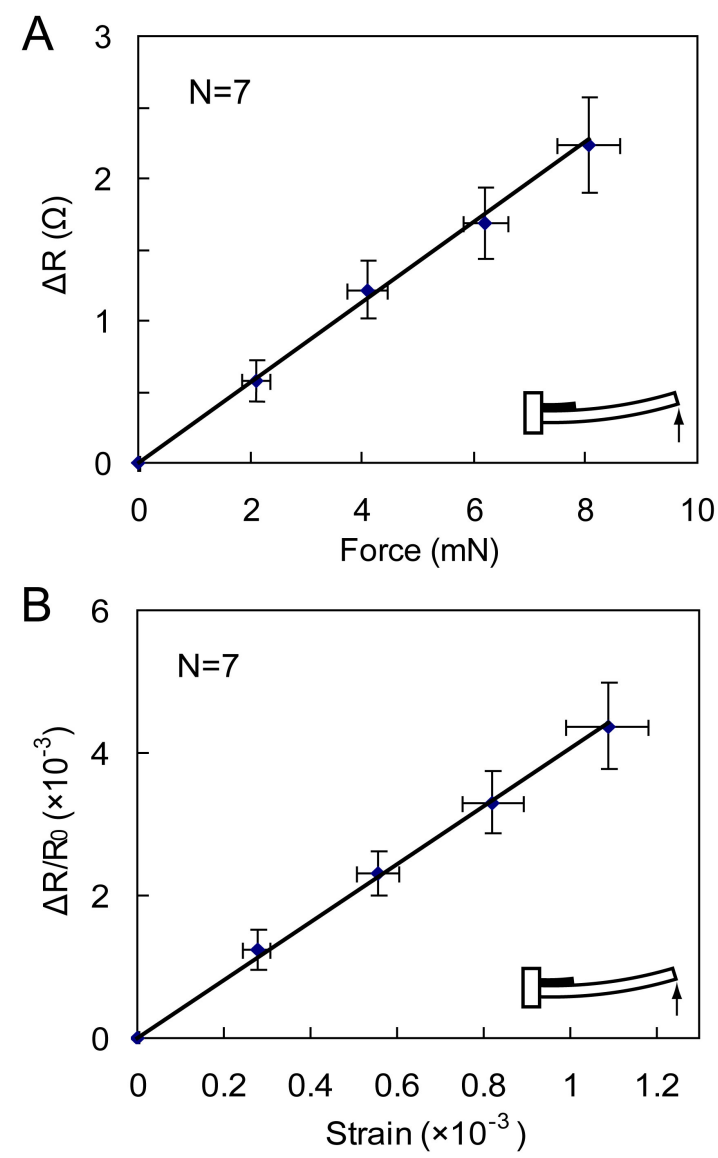
experimental results in the rest of this paper were collected by operating the sensor in the compressive mode.

We calculated the gauge factor of the piezoresistive sensor, which is defined as the ratio of relative change in resistance of the resistor $\left(\Delta R / R_{0}\right)$ to the applied mechanical strain $(\varepsilon)$. A higher gauge factor indicates higher sensitivity for the sensor. Fig. 2B shows that the relative resistance changed linearly with the applied strain; the gauge factor was 4.1.

We also quantified the reproducibility of the performance of paper-based sensors with and without silanization by calibrating a sensor seven times. Fig. S5 shows the calibration data collected from an un-silanized sensor (Fig. S5A) and a silanized sensor (Fig. S5B). The silanized sensor generated more reproducible output than the un-silanized sensor, probably because the silanization of paper surface minimizes the effect of environmental humidity on the output (change in resistance) of the sensor. The relative variation of output of the silanized sensor is within $5 \%$.

\section{Folding of the paper cantilever}

One characteristic of the paper not shared by silicon or quartz is that paper can be folded to form three-dimensional structures; this characteristic makes it straightforward to increase the stiffness of the paper sensor while keeping it light. We demonstrated the folding of a paper cantilever beam with the same dimensions as the ones we tested above. As shown in Fig. 3A, we cut dashed fold lines on the paper cantilever beam using a laser cutter, and folded the beam to form a wedge-shaped structure (Fig. 3B) that is stiffer than the un-folded part of the beam. This folding enhanced the stiffness of the cantilever beam without increasing the weight of the beam. Fig. 3C shows the force-deflection curves of the folded and un-folded beams $(\mathrm{N}=7)$; 
Fig. 3 Folding of the paper cantilever beam increases stiffness of the beam and the sensitivity of the sensor. (A) Schematic diagram of a paper cantilever beam with fold lines cut by a laser cutting. (B) A photograph of a folded sensor with a wedge-shaped structure. (C) Force-deflection curves of the folded and un-folded beams. The stiffness $(2.8 \mathrm{mN} / \mathrm{mm})$ of the folded beams is $40 \%$ higher than that $(2.0 \mathrm{mN} / \mathrm{mm})$ of the un-folded beams. (D) Calibration plots of output (change in resistance) of the sensor as a function of input (force applied to the free end of the beam) of the sensor. Due to the fact that the folding of the beam concentrates mechanical strain on the carbon resistor, the sensor with a folded beam produced higher sensitivity $(0.36 \Omega / \mathrm{mN})$ than the sensor with an un-folded beam $(0.29 \Omega / \mathrm{mN})$. 
Fig. 3
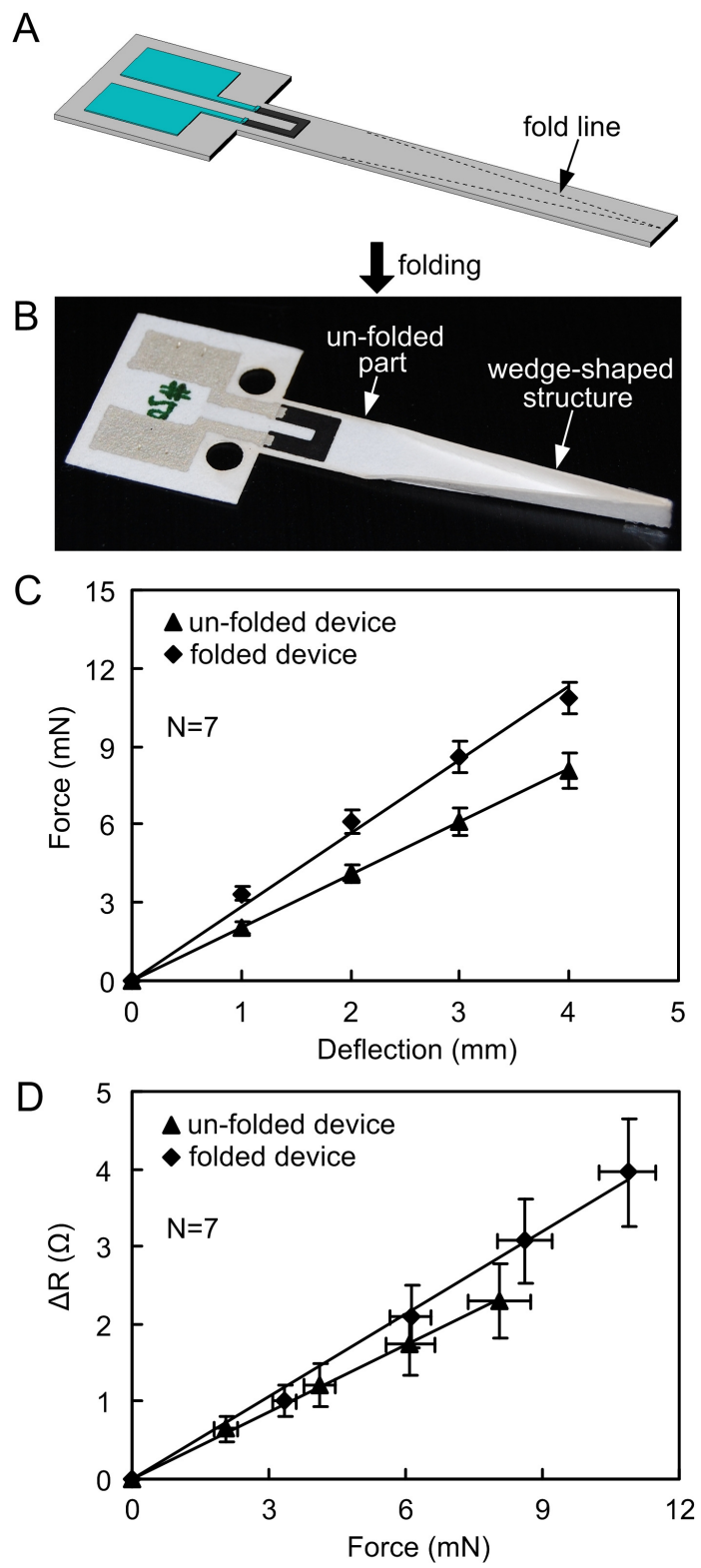
the stiffness $(2.8 \mathrm{mN} / \mathrm{mm})$ of the folded beam is $40 \%$ higher than that $(2.0 \mathrm{mN} / \mathrm{mm})$ of the unfolded beam. The folding of the cantilever beam also concentrated mechanical strain on the carbon resistor, and thus increased the sensitivity of the sensor. Fig. 3D shows calibration plots of the output (change in resistance) of the sensor as a function of the input (force applied to the free end of the beam) to the sensor. The sensitivity of the folded sensors was $0.36 \Omega / \mathrm{mN}$, which is $24 \%$ higher than the sensitivity $(0.29 \Omega / \mathrm{mN})$ of the un-folded sensors.

\section{Monolithic integration of a signal processing circuit onto the paper device}

In order to convert the change in resistance of the sensor into a more readable electrical signal (voltage), a Wheatstone bridge circuit (Fig. 4A) is commonly used for signal processing in MEMS piezoresistive sensing systems. There are two ways in conventional MEMS to integrate signal-processing circuits with the MEMS sensor: (i) A two-chip approach, where a MEMS

device is mounted on a printed circuit board (PCB) on which the signal processing circuit is laid out; the electrical connection is achieved using wire-bonding. (ii) A monolithic approach, where a MEMS device and a conventional IC signal processing circuit (e.g., complementary metal oxide semiconductor - CMOS) are microfabricated on the same silicon chip (e.g., CMOSMEMS). The monolithic approach provides smaller chip footprint and much lower noise levels, but is more complicated to fabricate.

For our paper-based MEMS sensors, we developed a monolithic approach that integrated the whole Wheatstone bridge circuit with the paper-based sensor. This approach was inspired by a previous paper, ${ }^{12}$ which used paper as a flexible PCB for construction of circuits. We laid out connections of the entire Wheatstone bridge circuit (including the carbon resistor $\mathrm{R}_{\mathrm{s}}$ in Fig. 4B) by screen-printing silver ink on the base of the paper-based sensor, then gluing three surface- 
Fig. 4 Monolithic integration of a Wheatstone bridge circuit with the paper-based sensor. (A) Schematic diagram of a Wheatstone bridge circuit, where $\mathrm{R}_{\mathrm{s}}$ is the resistor with unknown resistance to be measured and $\mathrm{R}_{1}, \mathrm{R}_{2}$, and $\mathrm{R}_{3}$ are resistors with known resistance. (B) $A$ photograph of a Wheatstone bridge circuit laid out on the base of a paper-based force sensor. Electrical connections were screen-printed using silver ink to connect the four resistors. (C) Calibration plot of the output of the circuit as a function of the input to the sensor (force applied to the free end of the sensor beam). The solid line represents a linear fit to the experimental data with a regression equation: $y=1.4 x\left(R^{2}=0.995, N=7\right)$. 
Fig. 4
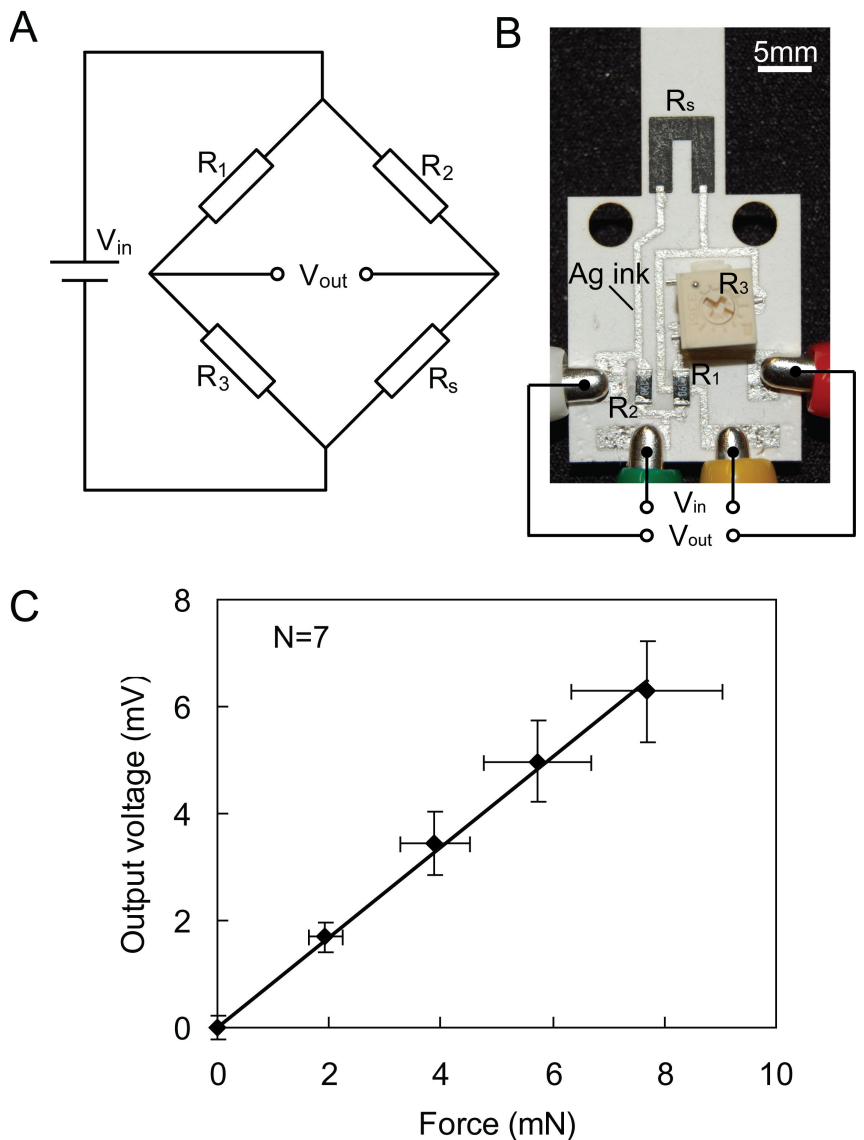
mount resistors $\left(\mathrm{R}_{1}, \mathrm{R}_{2}, \mathrm{R}_{3}\right)$ at appropriate locations, and finally 'soldering' them into the circuit using silver ink. $\mathrm{R}_{3}$ is an adjustable resistor that is used to initially balance the Wheatstone bridge.

Fig. 4C illustrates a calibration curve of the paper-based sensor with integrated Wheatstone bridge circuit. The resolution of the force measurement was improved to $120 \mu \mathrm{N}$ (corresponding to a voltage detection limit of $0.1 \mathrm{mV}$ ). The sensitivity of the sensor after integrating a wheatstone bridge circuit was $0.84 \mathrm{mV} / \mathrm{mN}$.

\section{Comparison of the paper-based sensor with a commercial silicon-based MEMS sensor}

Table 1 lists specifications of a commercial MEMS silicon force sensor (AE-801, Kronex Technology, specification data provided by the manufacturer) and our paper-based force sensor; both sensors are based on piezoresistive sensing. The commercial sensor is manufactured in silicon using standard microfabrication technology. Because the Young's modulus of the paper is much lower than silicon, the paper-based MEMS sensor has a low natural resonant frequency $(\sim 25 \mathrm{~Hz})$; this value indicates that the paper-based sensor could be used only for detection of low-frequency or static forces. The paper-based sensor has a lower force measurement range, resolution, and sensitivity than a commercial silicon-based sensor, but it has low cost, and requires only simple fabrication. In terms of fabrication and cost, the paper-based sensor may provide a simpler and less expensive solution for some force sensing applications than a siliconbased sensor.

\section{Applications}

After testing the device performance, we applied the paper-based force sensor to characterization of the mechanical properties of a soft material. Soft materials, such as polydimethylsiloxane (PDMS) and polyacrylamide (PAA), have been widely used for constructing micro devices ${ }^{13-16}$ and in mammalian cell culture. ${ }^{17}$ Mechanical properties (e.g., 
Table 1 Comparison of specifications of a commercial silicon-based MEMS sensor (AE-801, Kronex Technology) and our paper-based MEMS sensor.

\begin{tabular}{lll}
\hline Specifications & $\begin{array}{l}\text { Commercial silicon MEMS } \\
\text { sensor (AE-801, Kronex) }\end{array}$ & Paper-based MEMS sensor \\
\hline Sensing principle & Piezoresistive & Piezoresistive \\
Material & Silicon & Paper, carbon/silver inks \\
Beam size $(\mathrm{L} \times \mathrm{W} \times \mathrm{H})$ & $5 \mathrm{~mm} \times 1 \mathrm{~mm} \times 0.75 \mathrm{~mm}$ & $44.5 \mathrm{~mm} \times 7.7 \mathrm{~mm} \times 0.34 \mathrm{~mm}$ \\
Beam stiffness & $2000 \mathrm{mN} / \mathrm{mm}$ & $2 \mathrm{mN} / \mathrm{mm}$ \\
Natural frequency & $\sim 12 \mathrm{kHz}$ & $\sim 25 \mathrm{~Hz}$ \\
\hline Force range & $120 \mathrm{mN}$ & $16 \mathrm{mN}$ \\
Force resolution & $40 \mu \mathrm{N}$ & $120 \mu \mathrm{N}$ \\
Sensitivity & $2.5 \mathrm{mV} / \mathrm{mN}$ & $0.84 \mathrm{mV} / \mathrm{mN}$ \\
\hline Fabrication process & $>1 \mathrm{day}$ in cleanroom & $<1 \mathrm{hour}$ in laboratory \\
Device cost & $\$ 168 / \mathrm{device}($ commercial & $\$ 0.04 / \mathrm{device}$ (cost of \\
& price) & materials for a prototype)
\end{tabular}


Young's modulus) of soft materials are important in applications where the soft material serves as mechanical components (e.g., microfluidic valves/pumps ${ }^{18}$ and force sensing posts ${ }^{13-15}$ ) or cell culture substrates (the stiffness of which affects material's surface chemistry and cell behavior) ${ }^{17}$. Characterization of the mechanical properties of soft materials is based primarily on tensile testing and nanoindentation, both of which require access to expensive equipment. We demonstrated that our paper-based force sensor can be used to measure the Young's modulus of PDMS.

We prepared PDMS cantilever beams by cutting appropriately sized slabs from a sheet using a scalpel, and then controlled a paper-based force sensor to deflect the cantilever beam (Fig. 5A, the dimensions of the beams are summarized in Supplementary Table 1); during deflection, we measured the applied contact forces and resultant deflections of the cantilever beam. The Young's modulus of the PDMS was calculated using Equation 1. The time required to complete measurements with one cantilever beam was $<5$ minutes.

We tested three types of PDMS with different levels of cross-linking (mixing ratios (w/w) of the polymer base to the cross-linking agent: 5:1, 10:1, and 20:1). PDMS is supplied in two components: the un-cross-linked elastomer (polymer base) and the cross-linking agent. By varying the ratio $(\mathrm{w} / \mathrm{w})$ of the polymer base to the cross-linking agent, we changed the level of inter-chain cross-linking, and generated samples of PDMS with different stiffness (the stiffness decreases in the order: polymer $:$ cross-linker $=5: 1>10: 1>20: 1$ ). The experimental results (Fig. 5B) agree with the data in the literature. ${ }^{19,20}$

We also developed a paper-based weighing balance using the same piezoresistive sensing principle. As shown in Fig. 6A, paper-based force-sensing beams were used to tether a weighing plate and measure forces due to the gravity of an object placed on top of the weighing plate. 
Fig. 5 Mechanical characterization of PDMS using a paper-based force sensor. (A) Schematic diagram of the setup for measuring the Young's modulus of PDMS cantilever beams. A paperbased force sensor is controlled to contact and deflect a PDMS cantilever beam, during which the contact force and deflection of the PDMS beam are measured. The Young's modulus of the PDMS is calculated using a beam equation. The dimensions of the PDMS beams are summarized in Supplementary Table 1. (B) A plot of measured values of Young's modulus for PDMS with different mixing ratios (w/w: 5:1, 10:1, and 20:1) of the polymer base to the cross-linking agent. The values of Young's modulus in the plot are means of data from seven beams. 
Fig. 5
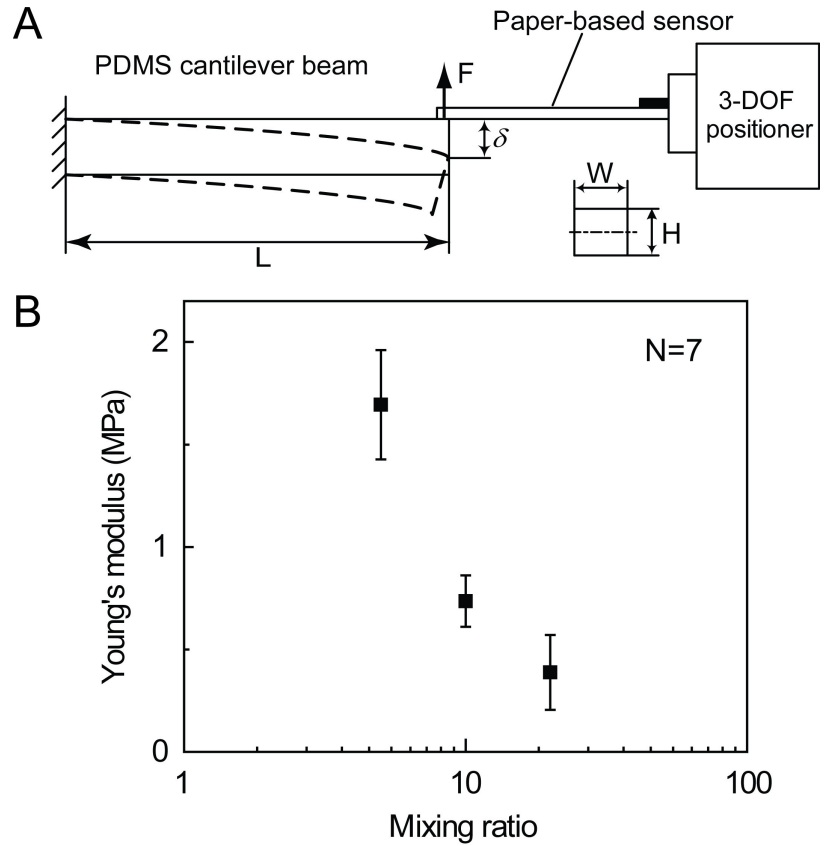
Fig. 6 A paper-based weighing balance. (A) A schematic side view of a paper-based balance where force-sensing beams with carbon resistors are used for tethering a weighing plate, and measuring the force due to gravity of a weight. (B) A photograph of the paper-based balance where four force sensing beams are involved. (C) Calibration plot of the resistance change from one sensing beam as a function of applied calibration weight. The solid line represents a linear fit to the experimental data with a regression equation: $y=0.26 x\left(R^{2}=0.993, N=7\right)$. 
Fig. 6
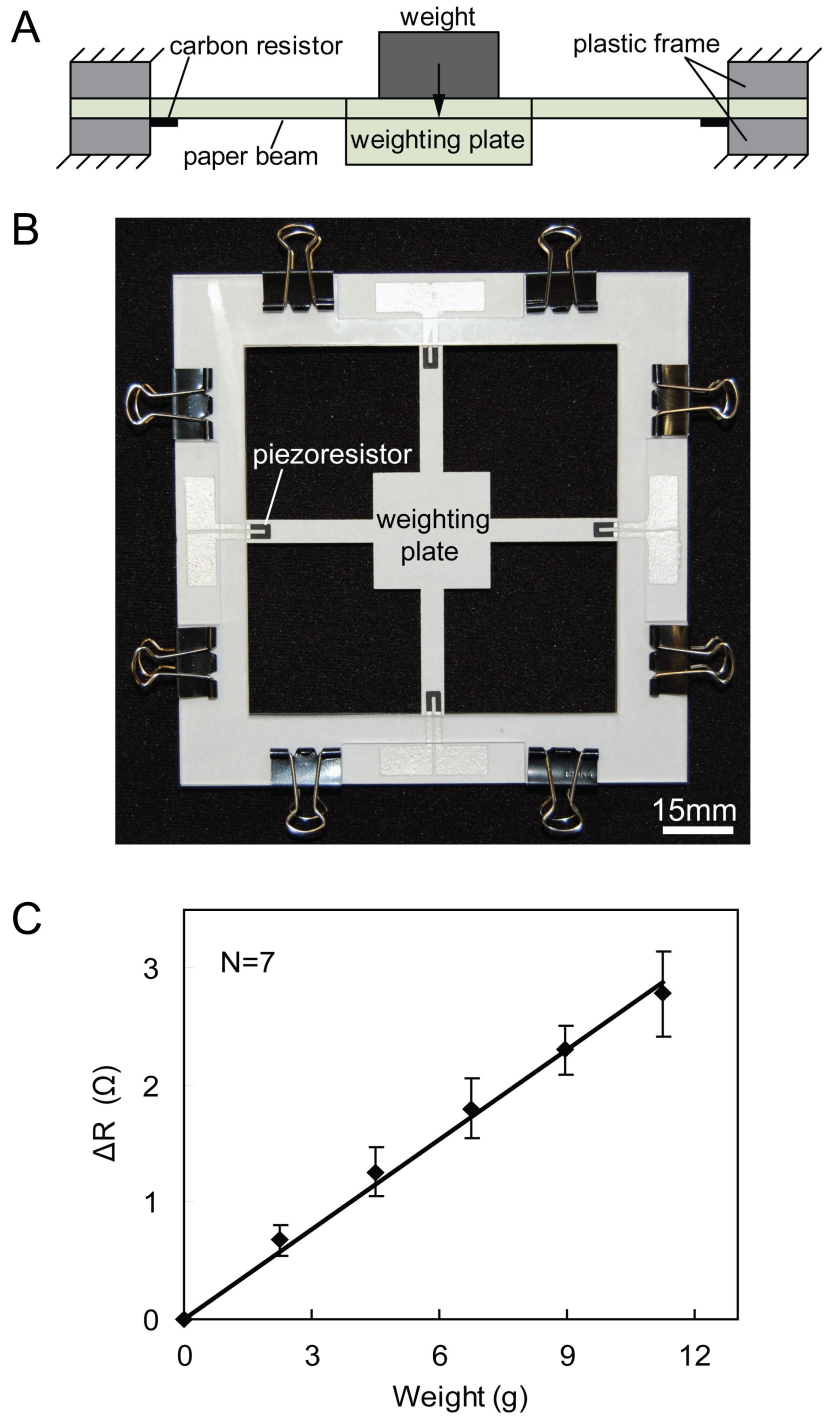
Fig. $6 \mathrm{~B}$ is the photograph of a balance prototype, where four force-sensing beams are involved. We calibrated the balance by measuring the change in resistance of the carbon resistor from one sensing beam as a function of the applied weight (Fig. 6C). The measurement range of the balance was $15 \mathrm{~g}$, and the resolution of the measurement was $390 \mathrm{mg}$.

\section{Conclusions}

We explored the feasibility of fabricating MEMS sensors using paper as the structural material, and developed paper-based piezoresistive force sensors. The use of paper for MEMS construction significantly simplifies the fabrication process relative to that used with silicon, and eliminates the requirement of cleanroom facilities. We also demonstrated folding of the paper cantilever beam of the sensor to increase the stiffness of the beam, and to improve the sensitivity of the sensor. The paper-based sensor presented in this paper is suitable for force sensing applications that requires moderate sensing capabilities and where the device cost is a major concern.

The paper-based MEMS technology has five advantages. (i) It represents a simple, fast, and low-cost solution for the problem of constructing low-cost MEMS devices. (ii) Paper, as the major material for device construction, is readily available, lightweight, and easy to manufacture (that is, there are highly developed technologies for cutting and folding it). (iii) Paper can be folded into three-dimensional structures with high stiffness and anisotropic responses but light weight; there is no corresponding capability in silicon. (iv) Manufacturing of paper-based MEMS devices has the potential to be fairly simple and to involve low-cost tooling; prototyping can be carried out using very simple tools, does not require access to cleanrooms, and has the potential for mass production (by automatic paper cutting and screen printing). (v) Paper can also be used as a substrate for laying out simple electrical circuits, and permits electrical circuits for 
signal processing to be readily integrated with the paper-based sensor to form monolithic paperbased chips.

The paper-based MEMS force sensor has two limitations. (i) It has lower sensing performance (i.e., measurement range, resolution, and sensitivity) than the silicon-based force sensor. (ii) Because paper has a lower Young's modulus (2 GPa) than silicon (130-170 GPa), it has a low natural resonant frequency $(\sim 25 \mathrm{~Hz})$, and is therefore limited to low-frequency or static measurement of forces.

\section{Acknowledgements}

This work was supported by the Bill \& Melinda Gates Foundation (\#51308), the MF3

Micro/Nano Fluidics Fundamentals Focus Center, and postdoctoral fellowships from the Natural Sciences and Engineering Research Council of Canada (to X.Y.L. and X.J.L.) and NanoScience and Engineering Centre (to M.M.).

\section{References}

1. A. C. R. Grayson, R. S. Shawgo, A. M. Johnson, N. T. Flynn, L. I. Yawen, M. J. Cima and R. Langer, Proc. IEEE, 2004, 92, 6-21.

2. C. Liu, Adv. Mater., 2007, 19, 3783-3790.

3. K. D. Wise, Sens. Actuat. A-Phys., 2007, 136, 39-50.

4. K. E. Petersen, Proc. IEEE, 1982, 70, 420-457.

5. A. W. Martinez, S. T. Phillips, M. J. Butte and G. M. Whitesides, Angew. Chem. Int. Ed. Engl., 2007, 46, 1318-1320.

6. A. W. Martinez, S. T. Phillips, G. M. Whitesides and E. Carrilho, Anal. Chem., 2010, 82, 310.

7. X. Li, J. Tian, T. Nguyen and W. Shen, Anal. Chem., 2008, 80, 9131-9134. 
8. R. Pelton, Trends Anal. Chem., 2009, 28, 925-942.

9. M. A. Nash, J. M. Hoffman, D. Y. Stevens, A. S. Hoffman, P. S. Stayton and P. Yager, Lab Chip, 2010, 10, 2279-2282.

10. N. Inagaki, Plasma surface modification and plasma polymerization, Technomic Publishing Company, Lancaster, Pennsylvania, 1996.

11. L. Gao and T. J. McCarthy, Langmuir, 2008, 24, 9183-9188.

12. A. C. Siegel, S. T. Phillips, M. D. Dickey, N. Lu, Z. Suo and G. M. Whitesides, Adv. Funct. Mater., 20, 28-35.

13. J. L. Tan, J. Tien, D. M. Pirone, D. S. Gray, K. Bhadriraju and C. S. Chen, Proc. Natl. Acad. Sci. U. S. A., 2003, 100, 1484-1489.

14. X. Y. Liu, Y. Sun, W. H. Wang and B. M. Lansdorp, J. Micromech. Microeng., 2007, 17, $1281-1288$.

15. Y. Zhao and X. Zhang, Sens. Actuator A-Phys., 2006, 125, 398-404.

16. X. Liu, R. Fernandes, A. Jurisicova, R. F. Casper and Y. Sun, Lab Chip, 2010, 10, 21542161.

17. X. Q. Brown, K. Ookawa and J. Y. Wong, Biomaterials, 2005, 26, 3123-3129.

18. M. A. Unger, H. P. Chou, T. Thorsen, A. Scherer and S. R. Quake, Science, 2000, 288, $113-116$.

19. D. Armani, C. Liu and N. Aluru, in Proc. IEEE Conf. Micro Electro Mechanical Systems, 1999, pp. 222-227.

20. A. Mata, A. Fleischman and S. Roy, Biomed. Microdevices, 2005, 7, 281-293.

21. Z. Nie, F. Deiss, X.Y. Liu, O. Akbulut and G. M. Whitesides, Lab Chip, 2010, 10, 31633169. 